\title{
New directions in the study of expertise in sport and exercise science
}

The aim of this special issue is to consider the factors that contribute towards expertise in sport, with contributions from scientists conducting research in the interrelated disciplines of perceptual-motor control and skill acquisition. There is a rich body of research in the area of sport expertise, much of which is well captured within the seminal expert-performance framework of Williams \& Ericsson (2005). This approach brings together research perspectives that span crosssectional experimental methods such as eye movement measures (Savelsbergh, Williams, van der Kamp, \& Ward, 2002), retrospective questionnaires that aim to capture the practice history of athletes (Ericsson, Krampe, \& Tesch-Römer, 1993), and intervention studies that have explored different methods aimed at enhancing the performance of expert populations (Farrow \& Abernethy, 2002). The special issue continues in this vein, with a collection of seven articles in which the works encompass both research studies and opinion pieces that focus on new perspectives, that have been proposed to stimulate original and future directions in the field of sport expertise research.

In the first article, Tedesqui, McCardle, Bartulovic, \& Young (2019), provide a critical perspective on selfreport methods previously used in extant survey-based research that has assessed, for instance, group differences in the practice activities of athletes of different skilllevels in sport expertise research. Primarily framed within the deliberate practice framework (Ericsson, et al., 1993), the authors highlight challenges in this research domain, particularly when discriminating between multiple, progressively skilled groups of athletes as well as the different types of practice activities taken. To counter this, the authors propose new approaches to improve the validity of how researchers can accurately assess differences between and within skill levels. Further, this submission describes a methodology that can be used to narrow athletes' estimation of time spent in a range of sport practice activities. This promising approach offers researchers the opportunity to enhance rigour in the implementation self-report methods when measuring the development of sport expertise.

The second article by Ng \& Button (2019), similarly considers challenges in the measurement of the development of expertise in sport, with a particular focus on the assessment of Fundamental Movement Skills (FMS), which are often seen as a precursor to the acquisition of more complex sport movements. Drawing on an ecological dynamics framework (Davids, Button, \& Bennett, 2008), the authors argue that commonly used assessment methods of FMS do not adequately capture the adaptation of motor competences that are realised during the achievement of a range of skilled movements. Further, the authors put forward a proposal of movement competence, which reflects the wide range of movement experiences that enable an individual to adequately explore and refine opportunities for action. In this respect, exploration of movement is recognised as an important requirement in the development of sport expertise. These proposals of $\mathrm{Ng}$ and Button are then considered relative to contemporary movement assessment tools that are available to practitioners and scientists, respectively.

The third article by Savelsbergh \& Wormhoudt (2019), similarly focusses on the development of motor competency as a foundation for the development of expertise. The manuscript provides an overview of the athletic skills model (ASM), which is an evidence-based framework for applied practitioners that enables the design and implementation of training interventions to enhance motor competence (physical literacy) as a foundation for expertise. More broadly, the authors outline how the ASM offers a framework for life-long physical activity participation, which maintains a balance between performance, fitness, and health for recreational and elite athletes. The authors outline how the ASM applies three "roads" to support the development of physical literacy: the concentric approach, the design of challenging environments, and the exploitation of skills transfer. Considered in isolation, or used in combination, the authors demonstrate how these "roads" can be used to support the development of functional variation in physical literacy, leading to an increased range of possibilities for skilled adaptation in sport expertise.

Seifert, Papet, Strafford, Coughlan, \& Davids (2019), also consider the relevance of the ASM as a framework in the longitudinal development of expertise, but as part of a broader ecological dynamics perspective (Davids, et al., 2008), with a focus on the role of skill transfer. The authors present three pillars of an ecological dynamics framework 
that can be used in sport expertise research: starting with individual-environment coupling; adaptation of a complex dynamical (movement) system to interacting constraints; and the regulation of action with perception. These three pillars are drawn upon to outline the role of behavioural variability, exploration and the importance of general and specific skill transfer in the development of talent and expertise in athletes. Following the overview of theoretical concepts, the authors outline practical implications, encompassing the benefits of early diversification and unstructured play alongside variable and constraints-led practice in talent development programs.

Stone, Strafford, North, Toner, \& Davids (2019), also draw on an ecological dynamics perspective in the fifth article to provide a critical review of the use of virtual reality (VR) systems in sport expertise training. The authors caution that a scientific framework should be considered by applied practitioners and scientists to ensure that VR is appropriately used in practice and training. In particular, the authors outline that research and applications of VR in athlete development are still warranted, with examination of: (i) individualised training and assessment programmes; (ii) supporting exploration of variable and creative practice environments; (iii) and ensuring context dependent decision making, where technology permits.

Sixth, in an intervention study, Wilson, Dicks, Milligan, Poolton, \& Alder (2019) examine the relationship between action capabilities and movement initiation in a soccer anticipation task following either change of direction or small-sided games training. There was an overall improvement in counter movement jump performance for both training groups and this improvement was correlated with movement initiation in the soccerspecific anticipation test. The novel findings highlight that different training modes can potentially have a positive impact on anticipation performance. The analyses also identify that physical improvements and adjustments in anticipation may benefit from highlighting individual differences. Moreover, the authors suggest that further investigation of the relationship between training, anticipation, and action is warranted for the development of research and applied perspectives in expertise.

Finally, Fasold, Noël, Wolf, \& Hüttermann (2019), also provide a novel empirical contribution, considering the analysis of expertise and performance, in the interplay of sports officials (referees) as part of their collective performance as a team. The authors presented case-study data from two elite handball referees to assess how these officials coordinated their gaze during the decision-making process. Gaze fixations were analysed over an entire game, with results showing that handball referees' coordination of gaze behaviour may be less than optimal as $75 \%$ of fixations were allocated on the same aspects of game action at the same time. As such, the authors suggested that there were aspects of the game in which the officials were not adequately attending to all information. The authors considered these findings in the context of expertise research and highlight avenues for performance improvement.

Overall, the articles presented in this special issue illustrate the range of excellent research that is now apparent in the discipline of sport expertise. Across the respective articles, the authors pave the way for some exciting new empirical research directions that span different theoretical perspectives including deliberate practice (Ericsson, et al., 1993) and ecological dynamics (Davids, et al., 2008) frameworks as well as intervention methods that reflect burgeoning technological developments such as virtual reality as well as multidisciplinary training that is grounded in skill acquisition theory. Collectively the works therefore, not only form the basis of a guide for future research, but also hold important implications for applied practice.

\section{Matt DICKS* \\ Joseph O'HALLORAN Martina NAVARRO Guest editors}

University of Portsmouth, Portsmouth, United Kingdom

${ }^{*}$ Corresponding author: matt.dicks@port.ac.uk

\section{References}

Davids, K., Button, C., \& Bennett, S.J. (2008). Dynamics of skill acquisition: A constraints-led approach. London: Human Kinetics.

Ericsson, K.A., Krampe, R.T., \& Tesch-Römer, C. (1993). The role of deliberate practice in the acquisition of expert performance. Psychological Review, 100(3), 363.

Farrow, D., \& Abernethy, B. (2002). Can anticipatory skills be learned through implicit video based perceptual training? Journal of Sports Sciences, 20(6), 471-485.

Fasold, F., Noël, B., Wolf, F., \& Hüttermann, S. (2019). Coordinated gaze behaviour of handball referees: A practical exploration with focus on the methodical implementation. Movement \& Sport Sciences-Science \& Motricité. DOI: $10.1051 / \mathrm{sm} / 2018029$.

Ng, J.L., \& Button, C. (2019). Reconsidering the fundamental movement skills construct: Implications for assessment. Movement \& Sport Sciences-Science \& Motricité. DOI: $10.1051 / \mathrm{sm} / 2018025$.

Savelsbergh, G.J., \& Wormhoudt, R. (2019). Creating adaptive athletes: The athletic skills model for enhancing physical literacy as a foundation for expertise. Movement $\&$ Sport Sciences-Science \& Motricité. DOI: 10.1051/sm/2019004.

Savelsbergh, G.J., Williams, A.M., van der Kamp, J., \& Ward, P. (2002). Visual search, anticipation and expertise in soccer goalkeepers. Journal of Sports Sciences, 20(3), 279-287.

Seifert, L., Papet, V., Strafford, B.W., Coughlan, E.K., \& Davids, K. (2019). Skill transfer, expertise and talent development: An ecological dynamics perspective. Movement \& Sport Sciences-Science \& Motricité. DOI: 10.1051/sm/ 2019010.

Stone, J.A., Strafford, B.W., North, J.S., Toner, C., \& Davids, K. (2019). Effectiveness and efficiency of virtual reality designs 
to enhance athlete development: An ecological dynamics perspective. Movement $\&$ Sport Sciences-Science $\&$ Motricité. DOI: $10.1051 / \mathrm{sm} / 2018031$.

Tedesqui, R.A., McCardle, L., Bartulovic, D., \& Young, B.W. (2019). Toward a more critical dialogue for enhancing selfreport surveys in sport expertise and deliberate practice research. Movement $\mathscr{G}$ Sport Sciences-Science $\mathcal{E}$ Motricité. DOI: $10.1051 / \mathrm{sm} / 2018027$.
Williams, A.M., \& Ericsson, K.A. (2005). Perceptual-cognitive expertise in sport: Some considerations when applying the expert performance approach. Human Movement Science, 24 (3), 283-307.

Wilson, R., Dicks, M., Milligan, G., Poolton, J., \& Alder, D. (2019). An examination of action capabilities and movement time during a soccer anticipation task. Movement $\&$ Sport Sciences-Science \& Motricité. DOI: 10.1051/sm/2019001.

Cite this article as: Dicks M, O'Halloran J, \& Navarro M (2018) New directions in the study of expertise in sport and exercise science. Mov Sport Sci/Sci Mot, 102, 1-3 